\title{
Ni-Mo-B 非晶态合金纳米颗粒的抗氧化性能研究
}

\author{
郑一雄 ${ }^{1,2}$ 姚士冰 ${ }^{1}$ 周绍民 ${ }^{1}$ \\ (1 厦门大学化学化工学院, 固体表面物理化学国家重点实验室, 厦门 361005 ; \\ 2 华侨大学材料科学与工程学院, 泉州 362011)
}

\begin{abstract}
摘要 在水溶液体系中用化学还原法制备 Ni-B 和 Ni-Mo-B 非晶态合金纳米颗粒. SEM 测试表明, 样品的颗粒 形貌呈球形或类球形, 平均粒径约 $10 \mathrm{~nm}$. XPS 分析结果表明, Ni-Mo-B 非晶态合金中锄元素主要以氧化态形成 于合金的表面, 并导致合金表面合金化硼原子浓度显著增加, 氧化态嗍 $\left(\mathrm{B}^{3+}\right)$ 的原子浓度显著减少, 氧的原子浓 度明显减少, 合金化镍的原子浓度显著增加, 氧化态镍 $\left(\mathrm{Ni}^{3}{ }^{3}\right)$ 的原子浓度显著减少. 因此, Ni-Mo-B 合金的抗氧 化性能显著高于 Ni-B 合金. XPS 谱图分析还表明, 在 Ni-B 和 Ni-Mo-B 非晶态合金中, 存在 $\mathrm{Ni}$ 和 B 的合金化物 $\mathrm{Ni}_{2} \mathrm{~B}$, 其中 $\mathrm{B}$ 失去部分电子, 而 $\mathrm{Ni}$ 则富余电子. $\mathrm{Ni}_{2} \mathrm{~B}$ 的氧化产生副产物 $\mathrm{Ni}_{2} \mathrm{O}_{3}$ 和 $\mathrm{B}_{2} \mathrm{O}_{3}$.
\end{abstract}

关键词: Ni-Mo-B 非晶态合金, 纳米颗粒，抗氧化, XPS 中图分类号: 0614

非晶态合金是一种热力学亚稳材料, 具有长程 无序、短程有序的特殊结构. 合金纳米粒子具有比 表面积大、表面原子比例及表面能高等特点.非晶 态合金纳米粒子兼备非晶态和纳米粒子的特性, 其 短程有序仅出现在第一原子近邻尺度内, 表面原子 配位高度不饱和, 从而具有许多特殊的功能, 在高 密度磁记录材料、铁流体、催化等领域都有重要应 用 $[1-4]$ 。

在水溶液体系中以化学还原法制备 Ni-B 非晶 态纳米粒子具有操作简单、条件易于控制及组成可 控等优点, 在催化加氢过程中, 其初始活性和选择 性优于传统工业上使用的 Raney 镍, 因而近年来受 到广泛关注, 并致力于将其发展成为新一代工业催 化剂 ${ }^{[5-8]}$. 但是, 目前所制备的 Ni-B 非晶态纳米粒 子在空气中极易氧化甚至着火, 从而降低催化活 性 ${ }^{[1,9-11]}$. 研究其抗氧化性能是其产业化道路上需 解决的一个关键技术。借助 $\mathrm{X}$ 射线光电子能谱技 术 $(X P S)$ 人们发现, 在 Ni-B 合金中, 合金化嗍失去 部分电子而合金化镍富余电子, 呈反电负性规律. 缺电子的合金化嗍可接受氧原子上的孤对电子而 优先氧化, 同时随着表面嗍的不断消耗, 体相中的 嗍会不断迁移至表面, 从而抑制表面镍活性位的 氧化 ${ }^{[11-12]}$. 因此, 增加合金化硼的含量是提高 $\mathrm{Ni}-\mathrm{B}$ 非晶态合金纳米粒子抗氧化性能的一个有效措施.
本文拟向 Ni-B 中引人第三种元素钼, 研究在水 溶液体系中用化学还原法制备 Ni-Mo-B 非晶态合 金纳米粒子的方法. 以 XPS 技术研究钿元素的掺 人对 Ni-B 非晶态合金纳米粒子抗氧化性能的影响.

\section{1 实验部分}

\section{1 样品制备}

将 $0.5 \mathrm{~mol} \cdot \mathrm{L}^{-1} \mathrm{Ni}(\mathrm{Ac})_{2}$ (用 $10 \% \mathrm{HCl}$ 调节成 $\mathrm{pH}=6$ ) 和 $0.5 \mathrm{~mol} \cdot \mathrm{L}^{-1} \mathrm{Na}_{2} \mathrm{MoO}_{4}$ (用 $10 \% \mathrm{HCl}$ 调节 成 $\mathrm{pH}=6$ ) 按一定体积比混合均匀, 置于冰盐水浴 中, 在激烈摚拌下, 由恒压漏斗逐滴滴加 $60 \mathrm{~mL}$ 、 $\mathrm{pH}=12$ 的 $1 \mathrm{~mol} \cdot \mathrm{L}^{-1} \mathrm{KBH}_{4}$ 水溶液, $20 \mathrm{~min}$ 内加完. 继续摚拌反应 $10 \mathrm{~min}$, 精确控制反应温度 $(0 \pm 1)$ ${ }^{\circ} \mathrm{C}$ 及恒定的滴加速度 $\left(3 \mathrm{~mL} \cdot \mathrm{min}^{-1}\right)$. 反应结束后, 离心分离黑色产物, 用蒸馏水洗涤数次到 $\mathrm{pH}=7.0$, 再用无水乙醇脱水, 最后真空干燥。制得的粒子暴 露于空气中会起火, 引人适量空气控制真空度为 $1.33 \times 10^{4} \mathrm{~Pa}$, 钝化处理 $8 \mathrm{~h}$, 将钝化后的粉末样品 压成厚度约 $1 \mathrm{~mm}$ 的薄片, 保存在无水乙醇中备用. 表 1 为制备不同 Ni-Mo-B 非晶态合金纳米颗粒样 品所量取的 $\mathrm{Ni}(\mathrm{Ac})_{2}$ 溶液和 $\mathrm{Na}_{2} \mathrm{MoO}_{4}$ 溶液的体积.

\section{2 合金组成分析和表征}

样品的体相组成由扫描电子显微镜 (SEM, LEO-1530, 德国)上所附带的 X 射线能量色散谱仪 
表 $1 \mathrm{Ni}-\mathrm{Mo}-\mathrm{B}$ 非晶合金表面组成及使用 $\mathrm{Ni}(\mathrm{Ac})_{2}$ 和 $\mathrm{Na}_{2} \mathrm{MoO}_{4}$ 溶液的体积

Table 1 Surface composition of $\mathrm{Ni}-\mathrm{Mo}-\mathrm{B}$ amorphous alloy and volume of $\mathrm{Ni}(\mathrm{Ac})_{2}$ and $\mathrm{Na}_{2} \mathrm{MoO}_{4}$ solutions

\begin{tabular}{cccc}
\hline Samples & $V_{\mathrm{Ni}_{(\mathrm{Ac})} / \mathrm{mL}}$ & $V_{\mathrm{Na}_{2} \mathrm{MoO}_{4} / \mathrm{mL}}$ & Surface composition \\
\hline 1 & 40.0 & 0 & $\mathrm{Ni}_{28.9} \mathrm{~B}_{11.8} \mathrm{O}_{59.3}$ \\
2 & 37.6 & 2.4 & $\mathrm{Ni}_{28.6} \mathrm{Mo}_{8.9} \mathrm{~B}_{11.6} \mathrm{O}_{50.9}$ \\
3 & 32.8 & 7.2 & $\mathrm{Ni}_{29.6} \mathrm{Mo}_{11.6} \mathrm{~B}_{10.2} \mathrm{O}_{48.6}$ \\
\hline
\end{tabular}

(EDS, ISIS-300, 英国牛津公司) 测定, 加速电压为 $20 \mathrm{kV}$. 样品表面组成由 XPS 测定, 仪器为 PHI Quantum 2000 Scanning ESCA Microprobe(美国), 激 发源为 $\mathrm{Al}$ 靶, 带单色器, $h \nu=1486.6 \mathrm{eV}$, 功率为 25 $\mathrm{W}$, 光电子出射角为 $45^{\circ}, X$ 射线束斑为 $100 \mathrm{~nm}$, 能 宽为 $50 \mathrm{eV}$, 分析室基础压力 $\leqslant 1 \times 10^{-7} \mathrm{~Pa}$, 结合能 相对于污染 $\mathrm{Cl} s(284.6 \mathrm{eV})$. XRD 测试仪器为日本 RIGAKU 公司生产的 D/MAX-RC 转靶 X 射线粉末 衍射仪, 采用 $\mathrm{Cu}$ 靶 $K_{\alpha}$ 射线, 管电压 $35 \mathrm{kV}$, 管电流 $20 \mathrm{~mA}$, 以石墨单色器滤波, 扫描速度为 $8\left(^{\circ}\right) \cdot \mathrm{min}^{-1}$. 样品的形貌尺寸由德国 LEO-1530 扫描电子显微镜 表征.

\section{2 结果与讨论}

\section{1 样品组成}

用 $\mathrm{X}$ 射线能量色散谱仪 (EDS) 测得 Ni-Mo-B 合金体相钼和镍 $(\mathrm{Mo} / \mathrm{Ni})$ 的原子浓度比: 样品 2 为 0. 058; 样品 3 为 0. 19. XPS 测得 Ni-Mo-B 合金表面 钿和镍 $(\mathrm{Mo} / \mathrm{Ni})$ 的原子浓度比: 样品 2 为 0.31 ; 样 品 3 为 0.39 . 可见, Ni-Mo-B 合金表面钿元素含量 远高于体相钼元素的含量, 表明钼元素的掺人主要 存在于合金的表面. 由 $\mathrm{Ni} 2 p 、 \mathrm{Mo} 3 d 、 \mathrm{~B} 1 s$ 和 $\mathrm{O} 1 s$ 的 XPS 窄谱峰面积及其灵敏度因子计算得到 Ni-B 和 $\mathrm{Ni}-\mathrm{Mo}-\mathrm{B}$ 样品的表面组成: 样品 1 为 $\mathrm{Ni}_{28.9} \mathrm{~B}_{11.8} \mathrm{O}_{59.3}$; 样品 2 为 $\mathrm{Ni}_{28.6} \mathrm{Mo}_{8.9} \mathrm{~B}_{11.6} \mathrm{O}_{50.9}$; 样品 3 为 $\mathrm{Ni}_{29.6} \mathrm{Mo}_{11.6} \mathrm{~B}_{10.2} \mathrm{O}_{48.6}$. Ni-Mo-B 表面氧元素的含量明 显低于 Ni-B, 若扣除氧化态锄中氧的贡献, 则

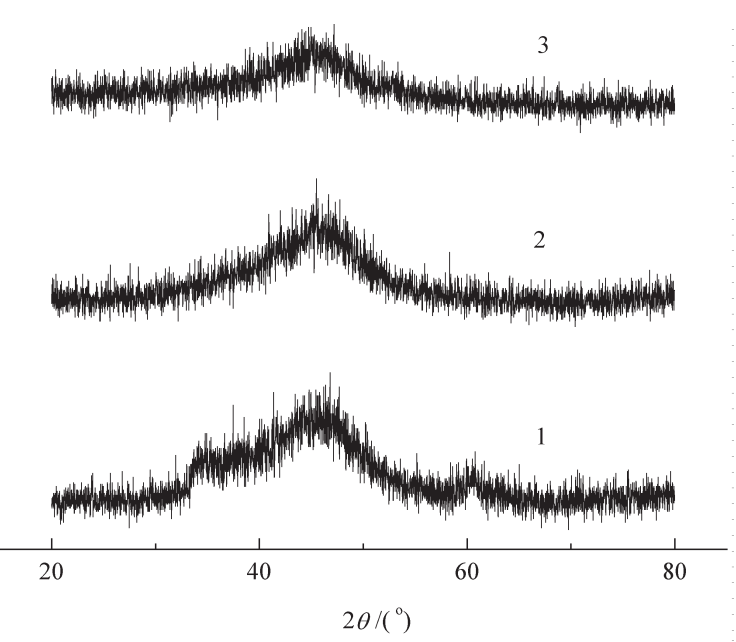

图 1 样品 $1 \sim 3$ 的 XRD 谱图

Fig. 1 XRD spectra of samples 1, 2 and 3

$\mathrm{Ni}-\mathrm{Mo}$-B 表面氧元素含量更低。可见掺人钿后, 合 金的抗氧化性能显著提高.

\section{2 非晶态结构的确定}

图 1 为样品 $1 \sim 3$ 的 XRD 谱图. 由图 1 可见, $\mathrm{Ni}-\mathrm{B}$ 和 Ni-Mo-B 样品的 XRD 谱图均在 $2 \theta=45^{\circ}$ 左 右出现宽化弥散峰, 这是 $\mathrm{Ni}$ 系非晶态合金的特征衍 射峰 ${ }^{[13-14]}$, 表明样品具有非晶态结构.

\section{3 样品的形貌尺寸}

图 2 为 Ni-B (样品 1) 和 Ni-Mo-B (样品 2、3) 的 SEM 照片, 样品的颗粒形貌是球形或类球形, 粒径 在 $10 \mathrm{~nm}$ 左右, 尺寸较均匀, 并呈现团聚状态.

\section{4 XPS 表征}

图 3 为 XPS 全谱分析图 $(0 \sim 1000 \mathrm{eV})$, 元素分 谱图表明 $\mathrm{O} 1 s 、 \mathrm{Ni} 2 p 3 / 2 、 \mathrm{Ni} 2 p 1 / 2 、 \mathrm{Mo} 3 d$ 和 Mo3 $p$ 分别在 $531.2 \mathrm{eV} 、 852.8 \mathrm{eV} 、 873.8 \mathrm{eV} 、 232.0 \mathrm{eV}$ 和 $398.4 \mathrm{eV}$ 处出现较强的谱峰. 全谱图中 B $1 s$ 峰强 度较弱未能显示出来. $\mathrm{Cl} s$ 污染峰出现在 284.6 $\mathrm{eV}$. 在 $700 \mathrm{eV}$ 处出现 $\mathrm{Ni}$ 的俄歇峰. 除了合金组成 外, 未发现其它杂质.

\section{4. 1 Mo 3d 窄谱分析}

样品 $2 、 3$ 的 Mo $3 d$ 窄谱扫描图及曲线拟合分
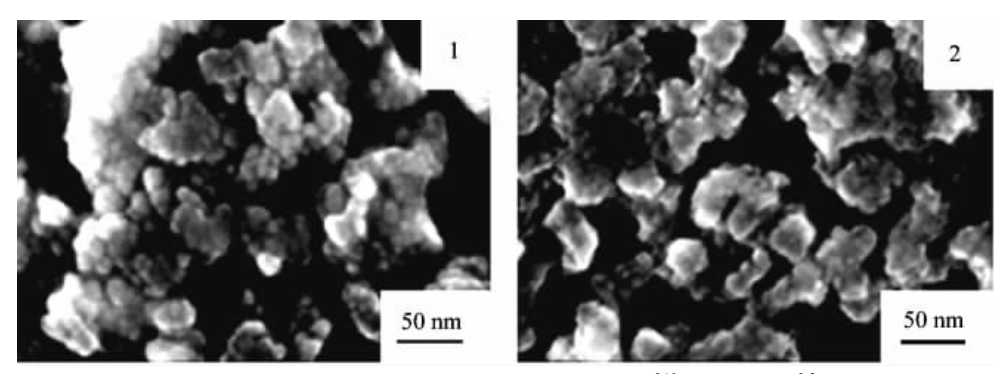

图 2 样品 1 3 的 SEM 图

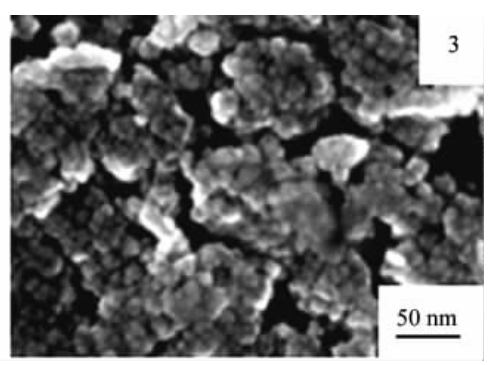

$50 \mathrm{~nm}$

Fig. 2 SEM graph of samples 1, 2 and 3 


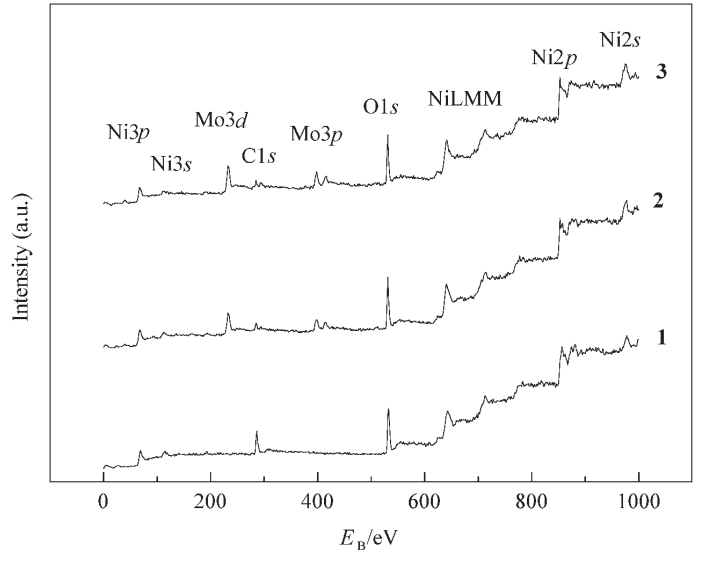

图 3 样品 $1 \sim 3$ 的 XPS 全谱图

Fig. 3 XPS survey spectra of samples 1, 2 and 3

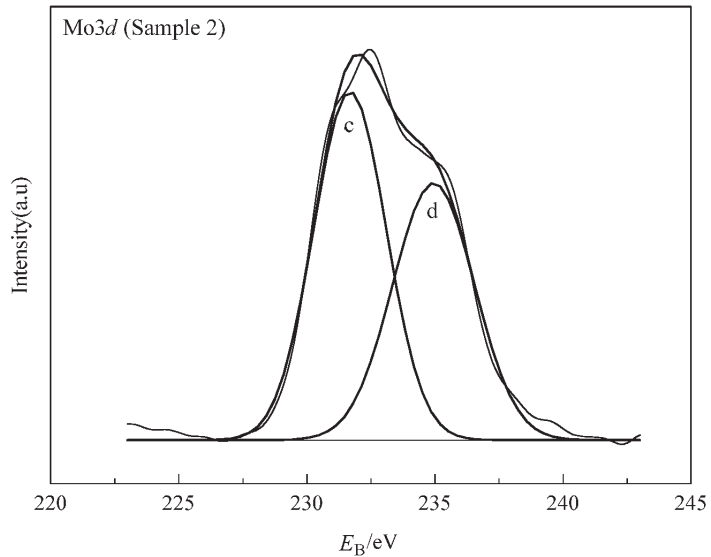

图 4 样品 2 的 Mo3 $d$ XPS 谱图及曲线拟合峰分离结果

Fig. 4 Mo3 $d$ XPS spectra and multipeaks curve fitting of sample 2

(c) $\mathrm{Mo}^{5+}(3 d 5 / 2)$; (d) $\mathrm{Mo}^{5+}(3 d 3 / 2)$

离谱峰结果见图 4、5, 拟合峰结合能列于表 2. 一般 而言, Mo 存在四种氧化态, 其结合能分别如下 ${ }^{[15-18]}$ : (1) $\mathrm{Mo}^{0}$ 的 $E_{3 d 5 / 2}$ 为 $228.0 \mathrm{eV}, E_{3 d 3 / 2}$ 为 $231.2 \mathrm{eV}$; $(2) \mathrm{Mo}^{4+}$ 的 $E_{3} d_{5 / 2}$ 为 $229.6 \mathrm{eV}, E_{3} d_{3 / 2}$ 为 $232.9 \mathrm{eV}$; $(3) \mathrm{Mo}^{5+}$ 的 $E_{3 d 5 / 2}$ 为 $231.5 \mathrm{eV}, E_{3} d 3 / 2$ 为 $234.7 \mathrm{eV}$; (4) $\mathrm{Mo}^{6+}$ 的 $E_{3 d 5 / 2}$ 为 $232.7 \mathrm{eV}, E_{3 d 3 / 2}$ 为 $236.0 \mathrm{eV}$. 由于 Mo 存在多种化学态, 而且结合能又相近, 反映 在 XPS 窄谱上的峰形较复杂, 且为重叠峰. 可以看 出, 样品 2 表面上钼元素主要以 $\mathrm{Mo}^{5+}$ 氧化态形式存

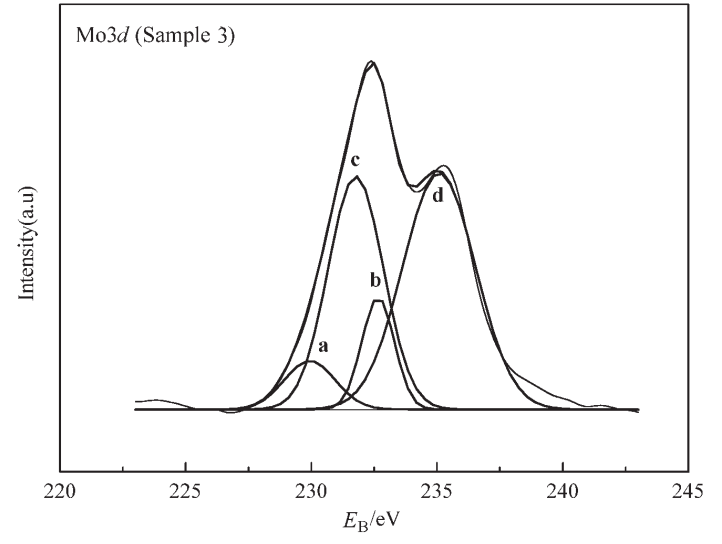

图 5 样品 3 的 Mo3d XPS 谱图及曲线拟合峰分离结果

Fig. 5 Mo3 $d$ XPS spectra and multipeaks curve fitting of sample 3

(a) $\mathrm{Mo}^{4+}(3 d 5 / 2)$; (b) $\mathrm{Mo}^{4+}(3 d 3 / 2)$;

(c) $\mathrm{Mo}^{5+}(3 d 5 / 2) ;\left(\right.$ d) $\mathrm{Mo}^{5+}(3 d 3 / 2)$

在. 样品 3 表面上钼元素主要以 $\mathrm{Mo}^{5+}$ 和 $\mathrm{Mo}^{4+}$ 氧化 态形式存在 .

\section{4.2 B 15 窄谱分析}

低分辨率下的 XPS 全谱分析图未能显示出B1 $s$ 谱峰, 而高分辨率下 $\mathrm{B} 1 s$ 的 XPS 窄谱上出现两个谱 峰(图 6), 表 2 给出了 $\mathrm{Bl} s$ 结合能峰值. $188.00 \mathrm{eV}$ 处的峰来自合金化嗍即 $\mathrm{Ni}_{2} \mathrm{~B}$ 中 $\mathrm{B}^{0[10-11]}$, 其结合能比 单质 $\mathrm{B}$ 的标准结合能 $(187.1 \mathrm{eV})^{[19]}$ 升高了 $0.9 \mathrm{eV}$, 表明在 Ni-B、Ni-Mo-B 合金中, $\mathrm{B}^{0}$ 失去部分电子使结 合能升高, 而合金化镍富余电子, $192.12 \sim 192.25 \mathrm{eV}$ 处的峰来自 $\mathrm{Ni}_{2} \mathrm{~B}$ 的氧化副产物 $\mathrm{B}_{2} \mathrm{O}_{3}{ }^{[11,20]}$. 由图 6 上谱峰的面积比可得 $\mathrm{B}^{0}$ 和 $\mathrm{B}^{3+}$ 的原子浓度比 $\left(\mathrm{B}^{0} /\right.$ $\left.\mathrm{B}^{3+}\right)$ : 样品 $1(\mathrm{Ni}-\mathrm{B})$ 为 0.14 ; 样品 $2(\mathrm{Ni}-\mathrm{Mo}-\mathrm{B})$ 为 0.37 ; 样品 $3(\mathrm{Ni}-\mathrm{Mo}-\mathrm{B})$ 为 0.49 . 可见, 掺人钼后, 合 金中 $\mathrm{B}^{0}$ 含量显著增加, $\mathrm{B}^{3+}$ 的含量显著减少, 并且锄 含量越大, $\mathrm{B}^{0}$ 含量越高, $\mathrm{B}^{3+}$ 含量越低.

\section{4. $301 s$ 窄谱分析}

图 7 为样品 $1 \sim 3$ 的 $\mathrm{O} 1 s$ 窄谱扫描图, 其峰值 结合能列于表 2 , 氧元素的原子浓度可见 2.1 节, 样 品 1(Ni-B) 的 O1 $s$ 结合能比其它样品 (Ni-Mo-B) 略 高, 氧元素的原子浓度明显高于其余样品。

\section{表 $2 \mathrm{~B} 1 s, 01 s$ 和 $\mathrm{Mo3} d$ 的结合能}

Table 2 Binding energy of B1 $s, \mathrm{O} 1 s$ and Mo3 $d$

\begin{tabular}{|c|c|c|c|c|c|c|c|}
\hline \multirow{2}{*}{ Sample } & \multicolumn{2}{|c|}{$E(\mathrm{~B} 1 s) / \mathrm{eV}$} & \multirow{2}{*}{$E(\mathrm{Ol} s) / \mathrm{eV}$} & \multicolumn{2}{|c|}{$E(\mathrm{Mo} 3 d 5 / 2) / \mathrm{eV}$} & \multicolumn{2}{|c|}{$E(\mathrm{Mo} 3 d 3 / 2) / \mathrm{eV}$} \\
\hline & $\mathrm{B}-\mathrm{Ni}$ & $\mathrm{B}-\mathrm{O}$ & & $\mathrm{Mo}^{4+}$ & $\mathrm{Mo}^{5+}$ & $\mathrm{Mo}^{4+}$ & $\mathrm{Mo}^{5+}$ \\
\hline 1 & 188. 13 & 192.25 & 531.75 & & & & \\
\hline 2 & 188. 00 & 192. 12 & 531.25 & & 231.69 & & 234.95 \\
\hline 3 & 188.00 & 192.25 & 531.00 & 229.93 & 231.77 & 232.66 & 235.08 \\
\hline
\end{tabular}




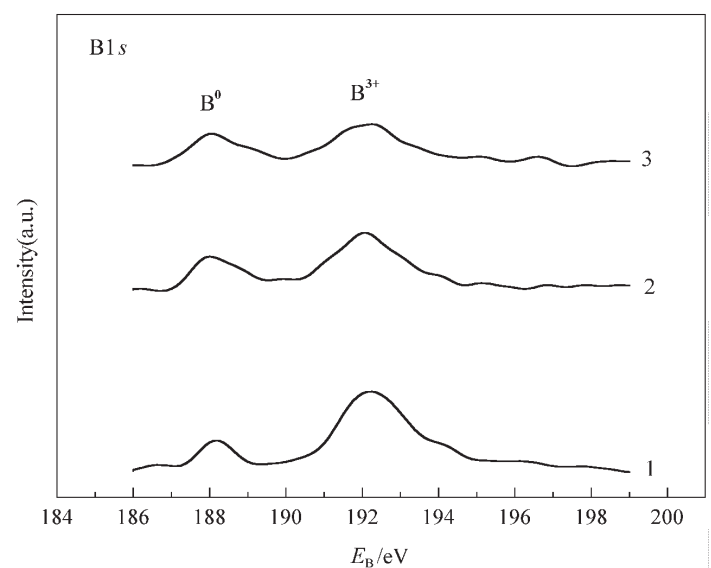

图 6 样品 1 3 的 B1 sXPS 谱图

Fig. 6 B1 $s$ XPS Spectra of simples 1, 2 and 3

\section{2. $4.4 \mathrm{Ni} 2 p$ 窄谱分析}

图 8 为样品 $1 \sim 3$ 的 Ni2 $p$ 的窄谱扫描图, 其 $\mathrm{Ni} 2 p 3 / 2$ 和伴峰的结合能及差值 $\Delta E$ 列于表 3 . 金 属镍、 $\mathrm{Ni}_{2} \mathrm{~B} 、 \mathrm{NiO}$ 和 $\mathrm{Ni}_{2} \mathrm{O}_{3}$ 的结合能值系文献数 据 ${ }^{[10,19,21-22]}$, 并列于表 3 作为比较. 图 8 中 852.50 $\sim 852.75 \mathrm{eV}$ 处的谱峰来自合金化镍, 与金属镍的 $\mathrm{Ni} 2 p 3 / 2$ 结合能 (852.95 eV) 比较, 其结合能有所下 降, 说明在 Ni-B (样品 1)、Ni-Mo-B (样品 $2 \sim 3$ ) 合金 中, $\mathrm{Ni}$ 获得部分电子使结合能降低, 与前面的分析 是一致的.

856. $13 \sim 856.38 \mathrm{eV}$ 处的伴峰来自于氧化态 镍 ${ }^{[11]}$, 从图 8 可见, 样品 $1(\mathrm{Ni}-\mathrm{B})$ 的 $\mathrm{Ni}$ 原子浓度明 显小于其它样品 (Ni-Mo-B), 而氧化态镍的原子浓 度明显高于 Ni-Mo-B 合金, 表明 Ni-Mo-B 合金的抗 氧化性明显高于 Ni-B 合金, Ni-Mo-B 合金表面有较 高的 $\mathrm{Ni}$ 原子浓度, 与前面的分析是一致的.

由于荷电位移的存在, 不可能准确测定

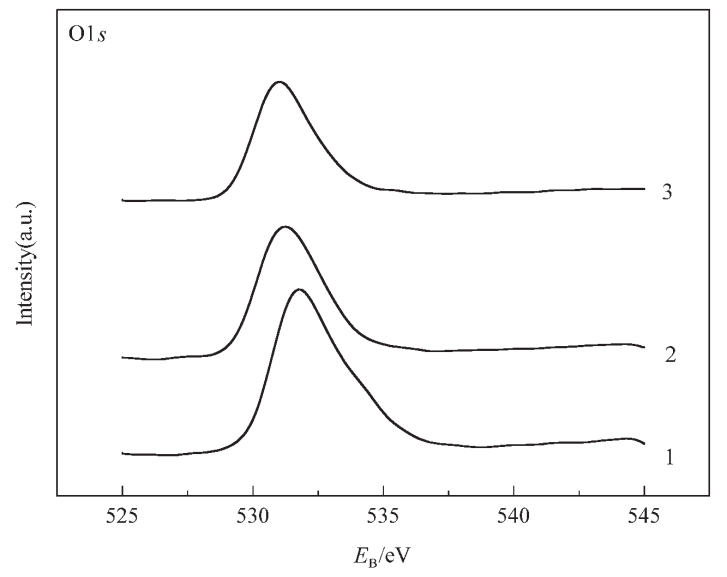

图 7 样品 $1 \sim 3$ 的 $01 s$ XPS 谱图

Fig. $701 s$ XPS spectra of samples 1, 2 and 3

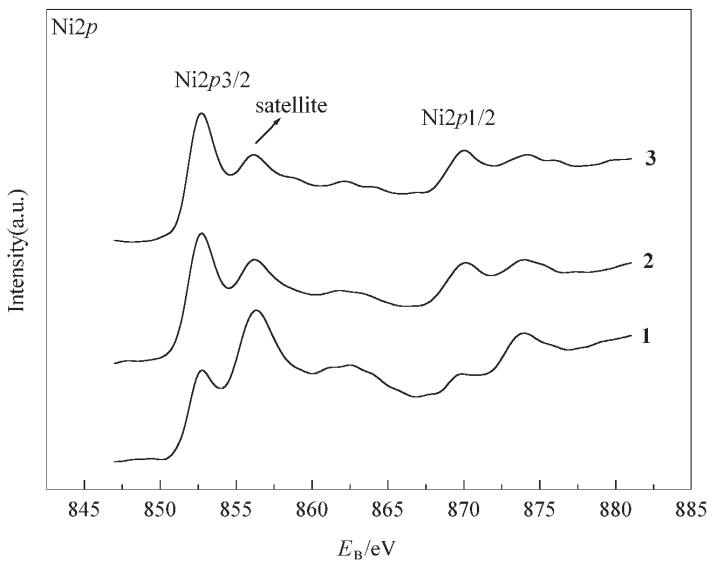

图 8 样品 $1 \sim 3$ 的 Ni2 $p$ XPS 谱图

Fig. 8 Ni2 $p$ XPS spectra of samples 1, 2 and 3

\section{表 $3 \mathrm{Ni} 2 p 3 / 2$ 和伴峰的结合能及其差值 $\Delta E$}

Table 3 Binding energies of Ni2 $p 3 / 2$ and satellite peaks, and their difference $\Delta E$

\begin{tabular}{cccc}
\hline Samples & $E(\mathrm{Ni} 2 p 3 / 2) / \mathrm{eV}$ & $E($ satellite $) / \mathrm{eV}$ & $\Delta E / \mathrm{eV}$ \\
\hline 1 & 852.63 & 856.25 & 3.62 \\
2 & 852.50 & 856.38 & 3.88 \\
3 & 852.75 & 856.13 & 3.38 \\
$\mathrm{Ni}_{2} \mathrm{~B}^{*}$ & 853.20 & 856.40 & 3.20 \\
$\mathrm{Ni} \mathrm{metal}^{*}$ & 852.95 & 858.70 & 5.80 \\
$\mathrm{NiO}^{*}$ & 853.75 & 855.55 & 1.80 \\
$\mathrm{Ni}_{2} \mathrm{O}_{3}{ }^{*}$ & 855.50 & & \\
\hline * data from ref. [10,19,21-22] & \multicolumn{5}{c}{}
\end{tabular}

$\mathrm{Ni} 2$ p3/2 结合能的绝对值. 另外, 将实验值与文献 的结合能直接比较也有一定误差. 因此, Ni2 $p 3 / 2$ 和伴峰的结合能差值也是有用的数据, 有助于识别 镍的氧化态 ${ }^{[10]}$. 从表 3 可以看出, $\mathrm{NiO}$ 的 $\Delta E$ 值明 显低于样品 $1 \sim 3$, 可将 $\mathrm{NiO}$ 排除, 图 7 上伴峰的结 合能值与 $\mathrm{Ni}_{2} \mathrm{O}_{3}$ 的 $\mathrm{Ni} 2 p 3 / 2$ 文献值接近, 可指认主 要来自 $\mathrm{Ni}_{2} \mathrm{O}_{3}{ }^{[11]}$. 样品 $1 \sim 3$ 的 $\Delta E$ 值明显低于金属 镍, 而与 $\mathrm{Ni}_{2} \mathrm{~B}$ 的 $\Delta E$ 值接近, 说明在 $\mathrm{Ni}-\mathrm{B}$ 和 $\mathrm{Ni}-\mathrm{Mo}-\mathrm{B}$ 合金中, 存在合金化镍 $\left(\mathrm{Ni}_{2} \mathrm{~B}\right)$, 与文献 [10] 认为 $\mathrm{Ni}-\mathrm{B}$ 合金中存在 $\mathrm{Ni}_{2} \mathrm{~B}$ 相符.

\section{3 结 论}

在水溶液体系中用化学还原法制得 Ni-B 和 Ni-Mo-B 非晶态纳米颗粒. XPS 的分析结果表明, 向 Ni-B 合金中引人钼元素后, 钿元素主要以氧化态 $\left(\mathrm{Mo}^{4+}\right.$ 和 $\left.\mathrm{Mo}^{5+}\right)$ 形式形成于合金的表面, 并导致合 金表面中 $\mathrm{B}$ 的原子浓度显著增加, $\mathrm{B}^{3+}$ 的浓度显著 减少,氧元素的原子浓度明显降低, $\mathrm{Ni}$ 的原子浓度 显著增加, $\mathrm{Ni}^{3+}$ 的浓度显著减少。因此, Ni-Mo-B 合 
金的抗氧化性能显著高于 Ni-B 合金。另外, 在 $\mathrm{Ni}-\mathrm{Mo}-\mathrm{B}$ 合金中, 存在 $\mathrm{Ni}$ 和 $\mathrm{B}$ 的合金化物 $\mathrm{Ni}_{2} \mathrm{~B}$, 其 中 $\mathrm{B}$ 失去部分电子, 而 $\mathrm{Ni}$ 则富余电子. $\mathrm{Ni}_{2} \mathrm{~B}$ 的氧化 产生副产物 $\mathrm{Ni}_{2} \mathrm{O}_{3}$ 和 $\mathrm{B}_{2} \mathrm{O}_{3}$.

\section{References}

1 Deng, J. F. ; Li, H. X. ; Wang, W. J. Catal. Today, 1999, 51: 113

2 Schwarz, J. A. ; Contescu, C. ; Contescu, A. Chem. Rev., 1995, 95: 477

3 Dorman, J. L. ; Fiorani, D. ; Tronc, E. Adv. Chem. Phys., 1997, 98: 283

4 Molnar, A. ; Smith, G. V. ; Bartok, M. Adv. Catal., 1989, 36: 329

5 Li, H. X. ; Li, H. ; Dai, W. L. ; Qiao, M. H. Appl. Catal. A: Gen., 2003, 238: 119

6 Satohiro, Y. ; Hiromi, Y. ; Takuzo, F. J. Chem. Soc. Faraday Trans. I, 1984, 80(6): 1435

7 Wang, X. G. ; Yan, W. S. ; Zhong, W. J. Chem. J. Chin. Univ., 2001, 22: 349 [王晓光, 国文胜, 钟文杰. 高等学校化学学报 ( Gaodeng Xuexiao Huaxue Xuebao), 2001, 22: 349]

8 Luo, H. S. ; Zhuang, L. ; Li, H, X. Journal of Molecular Catalysis, 2002, 16: 49 [骆红山, 庄 莉, 李和兴. 分子催化 (Fenzi Cuihua), 2002, 16: 49]

9 Legrand, J. ; Taleb, A. ; Gota, S. ; Guittet, M. J. ; Petit, C. Langmuir, 2002, 18: 4131
10 Glavee, G. N. ; Klabunde, K. J. ; Sorensen, C. M. ; Hadjipanayis, G. C. Langmuir, 1994, 10: 4726

11 Dai, W. L. ; Li, H. X. ; Cao, Y. ; Qiao, M. H. ; Fan, K. N. ; Deng, J. F . Langmuir, 2002, 18: 9605

12 Okamoto, Y. ; Nitta, Y. ; Imanaka, T. ; Teranish, S. J. Chem. Soc. Foraday Trans. I, 1979, 15: 2027

13 Corrias, A. ; Ennas, G. ; Musinu, A. ; Marongiu, G. ; Paschina, G. Chem. Mater., 1993, 5: 1722

14 Wang, M. H. ; Li, H. X. ; Wu, Y. D. ; Zhang, J. Materials Letter, 2003, 57: 2954

15 Brooks, A. R. ; Clayton, C. R. ; Doss, k. ; Lu, Y. C. J. Electrochem. Soc., 1986, 133: 2459

16 Clayton, C. R. ; Lu, Y. C. Corr. Sci., 1989, 29: 881

17 Lu, Y. C. ; Clayton, C. R. Corr. Sci., 1989, 29: 927

18 Yousif, K. M. ; Smish, B. E. ; Jeynes, C. J. Mater. Sci., 1996, 31: 185

19 Wagner, C. D. ; Riggs, W. M. ; Davis, L. E. ; Moulder, J. F. Handbook of X-ray photoelectron spectroscopy, Perkin-Elmer Corporation, Physical Electronics Division, Eden Prairie, MN, 1978

20 Petit, C. ; Taleb, A. ; Pileni, M. P. Adv. Mater., 1998, 10: 259

21 Beccaria, A. M. ; Wang, Y. Z. ; Poggi, G. Surf. Interface Anal., 1994, 21: 442

22 Davidson, A. ; Tempere, J. F. ; Che, M. ; Roulet, H. ; Dufour, G. J. Phys. Chem., 1996, 100: 4919

\title{
Study on Antioxidation of Nanosize Ni-Mo-B Amorphous Alloy
}

\author{
Zheng Yi-Xiong ${ }^{1,2} \quad$ Yao Shi-Bing $^{1} \quad$ Zhou Shao-Min $^{1}$ \\ ( ${ }^{1}$ State Key Laboratory of Physical Chemistry of Solid Surfaces, College of Chemistry and Chemical Engineering, Xiamen University, \\ Xiamen 361005; $\quad{ }^{2}$ College of Material Science and Engineering, Huaqiao University, Quanzhou 362011 )
}

\begin{abstract}
The nanosize Ni-B and Ni-Mo-B amorphous alloy powders were prepared by chemical reduction with $\mathrm{KBH}_{4}$ in aqueous solution. Their amorphous stuctures were determined by XRD. The SEM characterization demonstrates that all samples exhibit a spherical or sphere-like morphology with an average size of around 10 $\mathrm{nm}$. The XPS characterization shows that the molybodnum species in the Ni-Mo-B samples are mainly present on the surface of Ni-Mo-B alloy in the oxidized form. Compared with Ni-B alloy, the surface atomic content of alloying boron and alloying nickel of Ni-Mo-B alloy shows a considerable increase, the surface atomic content of oxidized boron, oxidized nickel and oxygen of Ni-Mo-B alloy shows a considerable decrease . Thus, XPS results have proven that the Ni-Mo-B amorphous alloy exhibits stronger antioxidation ability than the amorphous Ni-B alloy. XPS characterization also shows that $\mathrm{Ni}_{2} \mathrm{~B}$ is presented in the $\mathrm{Ni}-\mathrm{B}$ and $\mathrm{Ni}-\mathrm{Mo}-\mathrm{B}$ samples, and the alloying nickel is electron-enriched while the alloying boron is electron-deficient. $\mathrm{Ni}_{2} \mathrm{O}_{3}$ and $\mathrm{B}_{2} \mathrm{O}_{3}$ are byproducts of the $\mathrm{Ni}_{2} \mathrm{~B}$ oxidation.
\end{abstract}

Keywords: Ni-Mo-B amorphous alloy, Received: March 5, 2004; Revised: July 5, 2004.
Nanoparticle, Antioxidation, XPS 